\title{
La subordinación en el Glosario de términos gramaticales. Teoría, didáctica y su aplicación a la escritura*
}

\section{Subordination in the Glosario de términos gramaticales. Theory, didactics, and its application to writing}

\author{
Ángel J. Gallego \\ Universitat Autònoma de Barcelona \\ angel.gallego@uab.cat \\ ORCID ID: http://orcid.org/ 0000-0001-6352-458X
}

\section{Edita Gutiérrez-Rodríguez}

Universidad Complutense de Madrid

editagutierrez@ucm.es

ORCID ID: http://orcid.org/0000-0001-8631-4390

DOI: $10.17398 / 1988-8430.35 .2 .173$

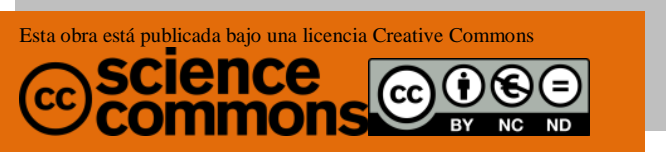

Fecha de recepción: 15/06/2021

Fecha de aceptación: 24/07/2021

OPEN $\odot$ ACCESS

Gallego, A. J., y Gutiérrez-Rodríguez, E. (2022). La subordinación en el Glosario de términos gramaticales. Teoría, didáctica y su aplicación a la escritura. Tejuelo, 35(2), 173-204.

Doi: https://doi.org/10.17398/1988-8430.35.2.173

\footnotetext{
* Este trabajo se ha beneficiado de las ayudas concedidas por el Ministerio de Economía y Competitividad (FFI2017-87140-C4-1-P, Gallego; PID2019-104405GB-I00, Gutiérrez-Rodríguez: PID2019-104405GBI00), AGAUR-Generalitat de Catalunya (2017SGR634, Gallego). Agradecemos a Xavier Fontich, a Ma José García Folgado y a Carmen Rodríguez Gonzalo su interés en nuestro trabajo y la invitación a participar en este volumen. Gracias también a dos revisores anónimos, cuyos comentarios han sido de gran ayuda de cara a la preparación de la versión final.
} 
Resumen: La gramática de la subordinación es una de las áreas más complejas de la gramática del español que los profesores de enseñanza secundaria tienen que explicar en las aulas. En este artículo se explica el análisis de la subordinación y, más concretamente, de la subordinación adverbial en la Nueva gramática de la lengua española y el Glosario de términos gramaticales. Se detallan las cuestiones en las que ambas obras coinciden y se justifican los casos en los que difieren. La idea que guía esta parte del artículo es que un buen conocimiento de la gramática por parte de los profesores permite hacer correcciones más específicas y claras, orientadas a la mejora de la escritura de los alumnos. En la segunda parte del artículo se ofrecen algunos ejemplos de cómo el tratamiento explícito de cuestiones gramaticales relacionadas con la subordinación puede ayudar a cumplir este propósito.

Palabras clave: enseñanza de la primera lengua; enseñanza de la gramática; enseñanza de la escritura; subordinación.

\begin{abstract}
The syntax of subordination is one of the most difficult subjects that a Spanish first language teacher has to address. In this article the analysis of subordination in the Nueva gramática de la lengua española and the Glosario de términos gramaticales are detailed, the two works are compared and the differences between them are explained and justified. The basic idea of this part of the article is that an explicit teaching of grammar can help students to improve their writing. In the second part of the article, we propose some exercises that show how knowledge of this area of grammar can be useful to this purpose.
\end{abstract}

Keywords: first language teaching; grammar teaching; teaching of writing; subordination. 


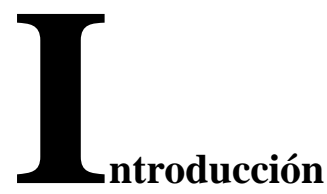

El objetivo de este artículo es doble. En primer lugar, presentaremos un análisis teórico de la subordinación que refleje los cambios introducidos en la Nueva gramática de la lengua española (NGLE, 2009), compararemos este análisis con la propuesta del Glosario de términos gramaticales (GTG, 2019) y, finalmente, explicaremos las diferencias entre una y otra obra. Nuestro objetivo es presentar un panorama claro de la subordinación dirigido a los profesores de secundaria y bachillerato. 
El tema de la subordinación es amplio y complejo, con muchas cuestiones sobre las que todavía hay debate entre los gramáticos. La existencia de ese debate no debe tomarse como algo negativo, sino como un síntoma de la dinamicidad del campo y del hecho de que existen muchos fenómenos lingüísticos que todavía no comprendemos en su totalidad. Desde nuestro punto de vista, que no los comprendamos no quiere decir que no deban conocerse ni que no deban discutirse en clase (ya sea de secundaria, bachillerato o universidad). Hemos tratado de reducir en la medida de lo posible las cuestiones que atañen al ámbito universitario para presentar un estado de la cuestión que resulte útil a los docentes de secundaria.

La idea que nos ha guiado en la elaboración de esta parte del artículo es que el conocimiento de gramática de un profesor de lengua ha de ser mucho más rico y sustantivo que aquello que explica a los estudiantes en el aula, como han defendido, entre muchos otros, Myhill, Jones y Watson (2013). Existe una idea generalizada, nos parece, de que debe darse una coincidencia total entre lo que el profesor de lengua sabe y lo que enseña; de tal manera, ante la posibilidad de estudiar tales o cuales contenidos que no va a presentar en el aula, la actitud de muchos profesores puede (y suele) manifestarse con reacciones como “Es necesario saber eso?”, "Se trata de contenidos demasiado complicados", "El estudiante no va a entenderlos", "No resultan útiles para escribir o leer mejor" entre otras. Creemos que tal actitud es equivocada, contraria, de hecho, a la que un profesional (de la materia que sea) debe manifestar en el ejercicio de su profesión. Solo sabiendo cuanto más mejor seremos capaces de superar las limitaciones que se dan en el aula (a través, en ocasiones, de los libros de texto) o los retos que nos pueden plantear los estudiantes con preguntas inesperadas. Solo sabiendo cuanto más mejor seremos también capaces de transmitir pasión e interés por nuestra asignatura. Basándonos en esa actitud, en estas páginas esbozamos los contenidos básicos sobre la subordinación que un profesor debería tener presentes, no tanto para llevarlos al aula, sino para poder decidir con conocimiento qué contenidos explicar y cómo hacerlo de manera creativa, reflexiva y competencial. 
En segundo lugar, queremos defender que un buen conocimiento de la gramática por parte de los profesores de lengua, en primer lugar, y de los estudiantes, como consecuencia de ello, puede ayudar a mejorar la competencia escrita. Empleando ejemplos relacionados con la subordinación, ofreceremos una muestra de cuál es el camino que creemos que hay que seguir para explotar la gramática en el desarrollo de la competencia escrita.

El artículo se organiza del modo siguiente: en el apartado 1 hablamos brevemente de la relación entre gramática y escritura; dedicamos el apartado 2 a la exposición teórica sobre la subordinación; finalmente, reservamos el apartado 3 a algunos ejemplos de aplicación didáctica de estos contenidos.

\section{1.- Relaciones entre gramática y escritura}

Una de las ideas que guía este trabajo es la convicción de que el conocimiento explícito de los principios gramaticales ayuda no solo a potenciar la capacidad argumentativa del estudiante (observación de datos, obtención de hipótesis, comprobación de predicciones, etc.), sino también a mejorar la competencia escrita. La gramática es uno de los conocimientos a los que se acude para escribir con propiedad. Como es sabido, el conocimiento lingüístico es solo una parte de las habilidades necesarias para redactar un texto. Por ejemplo, en la fase de planificación del texto (véase Cassany 1993 entre muchos otros) son importantes aquellas tareas que van más allá de lo lingüístico, como la selección de las ideas que se van a presentar y la organización de estas ${ }^{1}$. En cambio, el conocimiento lingüístico se vuelve fundamental tanto en la fase de textualización como en la de revisión de los textos; con "textualización" nos referimos tanto a aspectos que pertenecen a la oración (procesos de concordancia, relaciones semánticas entre palabras, uso de preposiciones, etc.) como a cuestiones vinculadas al

\footnotetext{
${ }^{1}$ Por esta razón, la tarea de aprender a redactar un texto no debería ser el objetivo de la clase de lengua únicamente, sino también del resto de las asignaturas. Los alumnos se beneficiarían ampliamente de practicar cómo se organiza la información que se les presenta en todas las asignaturas.
} 
discurso (relaciones fóricas, orden de constituyentes, introducción de referentes, estructuración de la información nueva/conocida, etc.).

Si consideramos el impacto del componente lingüístico en la producción de textos, el papel de las reglas gramaticales es central. Su control y conocimiento explícito permiten expresar de la manera más eficaz posible las relaciones lógicas entre las oraciones en el texto, ayudan a señalar la relevancia de determinadas ideas, permiten establecer un equilibrio entre lo dicho y lo nuevo, y permiten establecer las relaciones entre los distintos referentes discursivos, etc. En resumen, el conocimiento explícito de la gramática ofrece posibilidades de elección y de control del texto por parte del autor que le están vedadas al que no la domina. Pero, de nuevo, la gramática es solo uno de los factores que intervienen en la construcción de un texto, pues hay otras cuestiones lingüísticas que tienen que ver con el léxico, la semántica o la ortografía que son asimismo muy relevantes. Enmarcar el conocimiento gramatical en el espacio más amplio de la construcción de un texto puede permitir establecer relaciones entre los distintos componentes de la asignatura de lengua que, a menudo se presentan como compartimentos estancos.

Durante mucho tiempo (nos atrevemos a afirmar que, en algunos casos, incluso hasta la actualidad) se ha considerado que el conocimiento explícito de la gramática no servía para mejorar la competencia escrita. Hoy en día, sin embargo, numerosos estudios apuntan en la dirección contraria. Un buen ejemplo son los trabajos de Myhill y su equipo (Myhill et al., 2012, Myhill et al., 2013 entre muchos otros; en España, los trabajos de los grupos GREAL, GIEL y GrOC, por ejemplo, Fontich y Camps, 2015, Camps y Fontich, 2019 o Escandell, en preparación).

En Myhill et al. (2013) se señalan diferentes aspectos en los que el conocimiento gramatical de los alumnos permite hacer observaciones del profesor a un texto escrito que lo mejoren de forma sustancial. Si los alumnos, en cambio, no controlan una terminología gramatical básica y los conceptos asociados a ella, difícilmente el profesor podrá hacer ciertas correcciones que se demuestran útiles para la mejora del texto. 
¿Qué tipo de comentarios gramaticales pueden ayudar a un alumno a mejorar en la elaboración de un texto? Por ejemplo, un comentario explícito sobre la necesidad de añadir adjetivos a un texto descriptivo o, por el contrario, sobre el exceso de adjetivos; una observación sobre elecciones léxicas, como la escasa presencia de verbos modales (o al contrario) o de verbos de movimiento, por poner dos ejemplos muy distintos; una observación sobre la posición no marcada de los constituyentes y lo que implica avanzar un constituyente hacia la periferia izquierda oracional.

En resumidas cuentas, lo que planteamos aquí es la idea de que el conocimiento de la gramática (por parte de alumnos y profesores) y la guía del profesor en la corrección de un texto abre a los alumnos un abanico de elecciones y les ofrece un mayor control sobre el proceso de escritura. Por ejemplo, un conocimiento explícito sobre qué es la modalidad y qué posibilidades lingüísticas de realización de esta existen permite a los alumnos elegir entre un abanico de opciones para expresar de manera más sutil lo que se considera poco posible, posible, probable, etc. Como es sabido, el español expresa modalidad epistémica de muy diferentes maneras: a través de adverbios (seguramente, probablemente, quizá, tal vez, etc.), verbos modales (puede que, puede, etc.), adjetivos modales (seguro, posible, probable, etc.), verbos (saber, creer, etc., el modo verbal (cf. Quizá \{viene/venga\}) o los tiempos verbales (cf. Estará cansado / Se habrán ido al cine). El conocimiento explícito de los distintos procedimientos que ofrece el español para expresar modalidad -epistémica en este caso- permite hacer elecciones informadas y conscientes en el proceso de la escritura.

Sin embargo, como señalan Myhill et al. 2013 en su estudio, un factor importante en la mejora de la escritura de los estudiantes es el conocimiento gramatical del profesor. En el trabajo previo que estos autores hacen del conocimiento gramatical de los profesores, la mayoría de ellos son capaces de contestar correctamente solo a la mitad de las cuestiones gramaticales que se plantean. Uno de los problemas a los que se enfrentan los profesores de inglés como primera lengua es que han recibido una formación lingüística (y gramatical) muy escasa, lo cual 
repercute, naturalmente, en las posibilidades que tienen de emplear la gramática para la mejora de la producción escrita. En sus propias palabras:

\begin{abstract}
Subsequent stepwise regression modelling revealed that teachers' subject knowledge of grammar was a mediating factor $(p<0,050)$ in the success of the intervention, and equally, the analysis of the lesson observations and teacher interviews signalled the saliency of grammatical subject knowledge.
\end{abstract}

[tomado de Myhill et al. 2013: 81].

Como señalan Myhill et al. (2013: 87), uno de sus objetivos es crear, en las clases de lengua, oportunidades para discutir con los alumnos sobre la manera de escribir, las posibilidades que ofrece la lengua, etc. Sin embargo, este tipo de discusión genera comentarios y preguntas de los estudiantes sobre la gramática que ciertos profesores, con pocos conocimientos explícitos de gramática, no son capaces de abordar. Por ello, los profesores con menos confianza en sus conocimientos gramaticales tienden a cerrar cuanto antes esas discusiones, que pueden ser útiles para el desarrollo de la escritura en sus alumnos, evitando así los problemas gramaticales que creaban y que no son capaces de controlar. Si queremos ser capaces de explicar por qué no puede colocarse determinado signo de puntuación (no solo indicarlo, sino explicarlo), no podemos recurrir a argumentos vagos (y en ocasiones impresionistas) como "así se entiende mejor" o "no queda bien" o "afecta a la coherencia del texto". Es necesario ser más preciso, porque, de lo contrario, el estudiante que deba evitar ese problema no entenderá por qué debe hacerlo, lo cual le impedirá ver si el mismo razonamiento se aplica a otros errores que también comete.

En definitiva, el conocimiento explícito de la gramática puede ayudar a mejorar la práctica de la escritura. Sin embargo, para ello los profesores de primera lengua deben tener unos conocimientos gramaticales que a menudo no tienen. Es importante recordar que lo que sabe un profesor no es exactamente lo que lleva al aula (en nuestra opinión, un profesor debe saber mucho más de lo que enseña), y que el conocimiento gramatical influye en lo que los estudiantes aprenden. 


\section{2.- La subordinación en la NGLE y en el GTG: problemas terminológicos y conceptuales}

En este apartado examinaremos, en primer lugar, el análisis tradicional de la subordinación y los criterios que se emplean para diferenciar entre tres clases de subordinadas. A continuación, en el apartado 2.1, explicaremos cómo se analiza el grupo tradicional de las subordinadas adverbiales en la NGLE y cómo se reduce una parte de este grupo a las sustantivas y otra parte a las relativas. Por último, en el apartado 2.2, veremos el tratamiento del GTG de la subordinación y justificaremos los casos en los que esta obra difiere de la NGLE.

El análisis de la subordinación es uno de los temas más complejos a los que se enfrenta un profesor de secundaria. A la complejidad conceptual, se suma la complejidad terminológica en las aulas, que procede de la mezcla de sistemas lingüísticos (los más tradicionales, de base semántica, con los funcionales y los formales) en la tradición de la enseñanza de la lengua en España.

La clasificación más extendida de las oraciones subordinadas emplea una mezcla de criterios formales, funcionales y semánticos (cf. Brucart y Gallego, 2009, Brucart y Gallego, 2016, Areses y Pérez Jiménez, en preparación y referencias allí citadas). Por un lado, la noción de subordinación en sí misma implica una particular relación formal, de inclusión (o dominio, contención, etc.), entre dos constituyentes. Decir que una oración es subordinada implica afirmar que está dentro de un constituyente mayor, que puede ser típicamente el SV para muchas subordinadas sustantivas (Dijo que vendría), el SN para las relativas (Un niño que jugaba a la pelota), etc. En este sentido, supone un problema asignar el término oración subordinada a ciertas oraciones como las condicionales, las concesivas y algunas otras subordinadas que, cuando aparecen antepuestas, no se incluyen dentro de la principal ni son modificadores del SV, sino que mantienen con la oración principal una relación de concatenación que a veces se ha denominado interordinación (véanse Rojo 1978 o Rojo 1983). 
Por otro lado, la diferencia entre los tres tipos de subordinadas se establece básicamente con criterios funcionales. ${ }^{2}$ Se diferencia entre sustantivas o completivas, adjetivas o de relativo y adverbiales o circunstanciales, según las oraciones desempeñen las funciones típicas de un sintagma nominal (El juez decidió \{la pena/que iría a la cárcel\}), de un sintagma adjetivo (El libro \{interesantelque le interesó\} ( $^{3}$ o de un sintagma adverbial (Se fueron \{entonces/cuando salió el tren\}) respectivamente.

Las subordinadas sustantivas se definen en la NGLE: § 43.1a como aquellas que desempeñan las funciones características de los sustantivos o de los sintagmas nominales. Las oraciones sustantivas, por ello, son argumentales. En la definición de las subordinadas sustantivas también se incluye, sin embargo, un criterio formal, pues se señala que, desde el punto de vista de su estructura, las subordinadas sustantivas están formadas por la conjunción subordinante que y una oración, que constituye su término.

Nótese que los dos criterios anteriores no dan resultados equivalentes, pues tanto las oraciones de infinitivo como las interrogativas indirectas son sustantivas desde el punto de vista funcional, pero no desde el punto de vista formal: las oraciones sustantivas de infinitivo no están encabezadas por ninguna conjunción, ${ }^{4}$

${ }^{2}$ En ocasiones se emplea el término proposición subordinada con el sentido de oración subordinada. En la NGLE: $\S 43.1$ no se recomienda usar este término porque el concepto de 'proposición' recibe otro significado en la tradición lógica, que es el que se ha consolidado en los estudios lingüísticos. Los contenidos proposicionales son aquellos característicos de las oraciones, sean o no subordinadas. Estos contenidos aluden normalmente a hechos, situaciones o estados de cosas. El mismo criterio se sigue en la entrada de Oración en el GTG 2019.

${ }^{3}$ El paralelismo con los adjetivos no siempre se puede establecer. Los adjetivos son a menudo complementos predicativos y atributos, y pueden aparecer ante el nombre. Las relativas siempre aparecen en posición posnominal, no pueden ser atributo y desempeñan la función de complemento predicativo raramente (por ejemplo, en Los hay que siempre están cansados). Por otro lado, los adjetivos son raramente modificadores explicativos, frente a las relativas explicativas.

${ }^{4}$ Una manera de salvar esta objeción es considerar, como se ha hecho, que en las oraciones de infinitivo sí hay una proyección para la conjunción, que estaría vacía. De 
con la excepción de las interrogativas indirectas totales encabezadas por la forma si (No sé si ir contigo) ${ }^{5}$ en segundo lugar, las oraciones interrogativas y exclamativas indirectas no entrarían tampoco en la definición formal, pues no están encabezadas por una conjunción, sino por una palabra interrogativa o exclamativa. ${ }^{6}$ Mientras que las oraciones interrogativas indirectas se estudian en el capítulo 43, que se corresponde con la subordinación sustantiva, las oraciones de infinitivo se estudian en el capítulo 26, del infinitivo, junto con las relativas de infinitivo y otra serie de construcciones con infinitivo. Esta división en capítulos implica que se está privilegiando el criterio formal, en este caso, se emplea como criterio la forma no personal del verbo - o la ausencia de conjunción - para diferenciar una clase de oraciones.

En cuanto a las subordinadas adjetivas de la tradición funcional, en la NGLE se prefiere hablar de subordinadas de relativo, que se definen de manera formal como aquellas oraciones encabezadas por un elemento relativo, ya sea pronombre, adverbio o determinante. Esto implica, por un lado, incluir las llamadas adverbiales propias, como veremos más adelante, en el grupo de las relativas. Por otro lado, con este criterio saldrían de este grupo las construcciones de participio que modifican a los sustantivos dentro de un sintagma nominal y que en la tradición funcional se consideran adjetivas (El libro [editado por el escritor en Lugo] estaba listo para su distribución).

Por último, en el caso de las subordinadas adverbiales, más que la distribución propia de un adverbio, el criterio fundamental del acercamiento tradicional es la relación semántica de adjunción que se establece con el verbo (o con un segmento mayor para algunas

esta manera, todas las sustantivas constituirían una misma proyección, SComp en el modelo de la gramática generativa.

${ }^{5}$ Este elemento también se puede analizar como un adverbio interrogativo, en la medida en la que alterna con otros pronombres y adverbios interrogativos: No sé \{si/con quién/dónde/cómo\} ir.

${ }^{6}$ Las oraciones interrogativas y exclamativas indirectas se definen como aquellas que contienen pronombres o adverbios que fuerzan a seleccionar una opción entre varias (NGLE: § 43.7c). Nótese que con esta definición se agrupan las interrogativas indirectas con las directas (lo que parece correcto), pero estas oraciones quedarían fuera de la definición de subordinadas sustantivas que estamos manejando. 
adverbiales), pues, como es sabido, la mayoría de las subordinadas adverbiales no tienen la distribución de un adverbio ni se pueden sustituir por él. ${ }^{7}$

Hoy en día, una parte importante de los gramáticos considera que una clasificación que tome en cuenta criterios formales, es decir, la constitución interna de las oraciones, es preferible a una clasificación funcional. En concreto, de las tres clases definidas anteriormente, la clase tradicional de las subordinadas adverbiales es la más cuestionada porque se considera que no agrupa un conjunto uniforme de elementos desde el punto de vista formal. ${ }^{8}$ Veamos este aspecto con más detalle en la próxima sección.

${ }^{7}$ En realidad, las subordinadas adverbiales que sí se sustituyen por un adverbio, llamadas a veces adverbiales propias por ello, son las locativas ( $V a$ \{donde quiere/allí\}), temporales (Llegó \{cuando pudo/entonces\}) y modales (Vive \{como le gusta/asíf), que hoy en día se consideran relativas y no adverbiales, como veremos más adelante.

El término subordinada circunstancial es más amplio que el de adverbial y permite incluir en el grupo de las adverbiales algunas de las oraciones que no se pueden sustituir por adverbios, pero sí realizan la función de complemento circunstancial, como las causales o las finales. Sin embargo, incluso así se quedan fuera algunas oraciones que nunca se sustituyen por adverbios ni constituyen complementos circunstanciales, como las condicionales o las concesivas.

${ }^{8}$ Aunque emplear un criterio formal en lugar de funcional implica repensar también las otras dos clases. En primer lugar, hablar de subordinadas de relativo en lugar de subordinadas adjetivas supone reconsiderar el lugar de las oraciones de participio, que se consideran adjetivas porque realizan las funciones típicas de un adjetivo, pero no llevan relativo. Una parte de las oraciones de participio, las que son parte de una proyección nominal, se analizan en la tradición como adjetivas (el libro [traducido por el autor]), mientras que otras, entre ellas las cláusulas absolutas, se consideran adverbiales ([Una vez traducido el libro], se editó completamente). Igual ocurre con las subordinadas sustantivas de infinitivo, que no llevan conjunción completiva. Una solución que se ha propuesto para estos dos casos es la existencia de operador relativo nulo (para las oraciones de participio) y de una conjunción nula para las oraciones de infinitivo. 


\section{1.- La subordinación adverbial en la Nueva gramática de la lengua española}

Los problemas de análisis de las subordinadas adverbiales se parecen a los de la categoría adverbio, en el sentido de que bajo la etiqueta de subordinación adverbial se esconden elementos de naturaleza muy distinta, a los que no se puede dar un análisis unificado.

Por ello, la NGLE prescinde del concepto de subordinada adverbial y explica con detalle la manera en que se puede dar cuenta de los diferentes tipos de construcciones que tradicionalmente se engloban bajo esta etiqueta. ${ }^{9}$ La intuición más importante que se esconde tras la etiqueta necesariamente vaga de construcción que emplea la NGLE es que muchas de las secuencias que se consideran en la tradición oraciones subordinadas adverbiales no son ni siquiera oraciones.

\subsection{1.- Subordinadas adverbiales que son en realidad oraciones sustantivas}

El concepto de subordinada adverbial se rechaza en la mayoría de las teorías gramaticales actuales porque muchas de las construcciones consideradas oracionales no son en realidad oraciones, sino otro tipo de secuencias (sintagmas preposicionales o sintagmas adverbiales, principalmente) que contienen oraciones. Por tanto, el complemento verbal que establece una relación de adjunción con valor temporal, final o condicional con el verbo principal (llegar o llamar) no es en realidad una oración, sino, por ejemplo, un SAdv (Antes de que saliera el tren), un SP (Para que fueras con él) o un SN (La condición de que trabaje duro), todos con una oración en su interior:
a. Llegó [sAdv antes de [o que saliera el tren]]
b. Llamó [sp para [o que fueras con él]]

\footnotetext{
${ }^{9}$ La NGLE dedica el capítulo 45 a las construcciones comparativas, superlativas y consecutivas, el 46 a las construcciones causales, finales e ilativas, y el 47 a las construcciones condicionales y concesivas. En cambio, el capítulo 44 se denomina Las oraciones subordinadas de relativo y el 43 Las oraciones subordinadas sustantivas, estableciendo de esta manera un contraste claro entre unas y otras.
} 
c. Vendrá con [sN la condición de [o que trabaje duro]]

En segundo lugar, no está claro que las tradicionalmente consideradas subordinadas adverbiales tengan una naturaleza formal diferente de las oraciones subordinadas sustantivas o de las relativas (cf. Brucart y Gallego, 2009, Brucart y Gallego, 2016, Bosque y GutiérrezRexach, 2009). Es decir, se cuestiona que exista un tercer tipo de subordinación $\mathrm{y}$, por ello, se trata de reducir todo el grupo de las oraciones llamadas adverbiales en la tradición a los otros dos tipos: o bien se analizan como sustantivas, o bien como relativas. ${ }^{10}$ Vamos a ver a continuación cómo se ha llevado a cabo esta reducción para, posteriormente, revisar las oraciones más problemáticas para este análisis.

En los ejemplos de (1), las oraciones parecen tener la misma configuración y naturaleza que la subordinada de Juan dijo [o que venía]. El criterio de la tradición para considerarlas adverbiales es semántico y en realidad hace alusión a la relación semántica que se establece entre el predicado y un segmento mayor que la oración, como hemos visto. En la NGLE, estas oraciones se consideran subordinadas sustantivas de término de preposición, pues en estas construcciones alternan oraciones de infinitivo, oraciones flexionadas y SN (2a), igual que cuando una subordinada sustantiva es complemento directo de un verbo (2b):

\footnotetext{
${ }^{10}$ Más concretamente, Brucart y Gallego (2016) discuten dos maneras de subordinar: con un elemento subordinante como que, que puede tener realización fonética (Dijo que venía) o estar vacío (Quería ir con ella al cine), o bien con una palabra qu- interrogativos y relativos- que se origina en la oración subordinada y se desplaza al inicio de esta posteriormente. Esta clasificación situaría a relativas e interrogativas en un tipo y a las subordinadas sustantivas sin transformaciones en otro, dividiendo la clase de las subordinadas sustantivas. Nótese que la clase de las subordinadas sustantivas también se define con criterios funcionales, pues son aquellas oraciones que pueden realizar funciones típicamente nominales, como sujeto, complemento directo o término de preposición. La división que acabamos de presentar (oraciones introducidas por conjunción/por palabra $q u$-) tiene la ventaja de explicar las características comunes de relativas e interrogativas.
} 
Tejuelo, $n^{\circ} 35.2$ (2022), págs. 173-204. La subordinación en el Glosario de términos gramaticales...

(2) a. Llegó antes de \{que saliera el tren / salir el tren / la salida del tren $\}$

clase

b. Pidió \{que salieran antes / salir antes / la salida temprana \} de

El mismo análisis composicional de para que vengas se puede aplicar a una conjunción como porque. No tendríamos, por tanto, subordinadas adverbiales finales encabezadas por la locución conjuntiva para que o subordinadas adverbiales causales encabezadas por la conjunción causal porque, sino sintagmas preposicionales de sentido final $^{11}$ o causal. Eso simplifica (y unifica) considerablemente los análisis.

Se pueden analizar igual las oraciones que son término de locuciones prepositivas del tipo a fin de, por causa de, en caso de, a condición de o a pesar de. ${ }^{12}$ Por tanto, en una oración como Dijo que

${ }^{11}$ La misma reducción se puede hacer con todas las locuciones conjuntivas finales, por eso en la clasificación de conjunciones que aparece en la NGLE: $31.11 \mathrm{~h}$ no aparecen las conjunciones finales. No hay conjunciones de sentido final, sino preposiciones y locuciones prepositivas de significado final que toman subordinadas sustantivas como término. En el GTG, bajo el lema Conjunción final solo aparece el que de sentido final (Habla más, que te oigan todos). Sin embargo, incluso en este caso no está claro que existan diferentes formas que como conjunción causal (Vámonos ya, que estoy cansado), comparativo (Es más simpática que tú), etc. Otra opción es pensar que hay un solo que, la conjunción completiva, de manera que los distintos tipos de significado (causal, final, comparativo, consecutivo...) no se atribuirían a la conjunción, sino más bien a la interacción de varios factores, como la posición de la subordinada, el modo del verbo de la subordinada, etc., tal como se explica en el GTG en el lema Conjunción completiva.

${ }^{12}$ En los análisis escolares se califican como locuciones numerosos segmentos que no lo son (Pavón Lucero, 1999, Pavón Lucero, 2012, Brucart y Gallego, 2016, entre muchos otros). En primer lugar, secuencias como a condición de que, a pesar de que, a fin de que, etc. se consideran a veces locuciones conjuntivas que encabezan

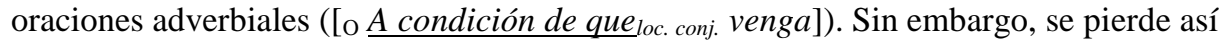
el siguiente paralelismo: a pesar de \{que venga/venir/su venida\}. Por ello, resulta más adecuado un análisis como locución prepositiva (no se puede analizar composicionalmente nada más porque el nombre -condición, pesar- no admite adjetivos o determinantes en estas locuciones). En segundo lugar, son segmentos composicionales, es decir, sintagmas preposicionales con sintagmas nominales como término, secuencias como con la (única) condición de que, con el (único) fin de, etc. Estos elementos introducen complementos de significado final, condicional, etc., pero 
llegaría pronto [a condición de que pudiera salir al día siguiente], la construcción entre corchetes no es una adverbial condicional encabezada por la locución conjuntiva a condición de que, sino un sintagma preposicional de sentido condicional (sentido que aporta el sustantivo condición), cuyo término es una oración subordinada sustantiva.

\subsection{2.- Subordinadas adverbiales que son en realidad relativas con antecedente implícito}

Otro grupo de oraciones, las llamadas en la tradición "adverbiales propias", se analizan en la NGLE: $\S 44.7$ como oraciones de relativo. Se privilegia aquí el criterio formal, que considera que toda oración encabezada por un relativo es una oración de relativo. Se evita así la duplicación categorial que aparece a menudo en los análisis escolares, que analiza donde en (3a) como un relativo y en (3b) como una conjunción. En estos análisis, se le asigna una función en la subordinada a donde en (3a), pero no en (3b), que se suele considerar únicamente un nexo:

(3) a. Fue al lugar donde quiso ir

b. Fue a donde quiso ir

Nótese que si en (3b) donde se considera una conjunción, no se puede señalar la relación que se establece entre el verbo de la subordinada ir y la forma donde, que es su complemento también en

son a todos los efectos sintagmas preposicionales con una oración subordinada sustantiva en el término del SP complemento del nombre. Por último, sí parecen locuciones conjuntivas las secuencias del tipo de siempre que, ahora que, ya que, etc., que no alternan con sintagmas preposicionales de término oracional o nominal (Siempre \{que viene/*de venir/*de su venida\} (para un análisis de estas secuencias, véase Brucart y Gallego, 2016, p. 172). En Pavón Lucero (2012) las oraciones con siempre se analizan como relativas (Siempre que viene trae un regalo / Siempre trae un regalo) y también la NGLE: 31.14h se inclina por este análisis. A favor de este análisis está que siempre puede modificarse con casi y negarse: No siempre que viene. Para un análisis de estas secuencias, véase Brucart y Gallego (2016: 172) 
(3b). ${ }^{13}$ Por todo ello, se acepta en la NGLE: 44.7 el análisis, originalmente planteado en Bello, que considera que las oraciones adverbiales propias son en realidad oraciones de relativo con un antecedente tácito: donde 'allí donde, en el sitio en el que', cuando 'entonces cuando, en el momento en que' y como 'así como, de la manera en que', tal y como queda reflejado en (4), donde el símbolo $\varnothing$ indica el antecdente tácito:
a. Voy $[\varnothing[$ donde quiero]]
b. Viaja [ $\varnothing$ [cuando puede]]
c. Lo hizo [ $\varnothing$ [como sabía]]

Hasta aquí hemos visto que hay razones empíricas para considerar que una parte de las subordinadas adverbiales son, en realidad, subordinadas completivas y otra parte son subordinadas de relativo con antecedente implícito y, por tanto, sintagmas adverbiales o preposicionales que contienen una relativa. Intentemos ver qué queda, una vez hecha esta redistribución, de la "subordinación adverbial".

\subsection{3.- Otras subordinadas adverbiales de difícil clasificación}

Hemos visto antes que hay un buen número, tradicionalmente consideradas adverbiales, que en realidad son sintagmas preposicionales con una oración subordinada sustantiva como término. En tal caso, las subordinadas ocupan una posición nominal (el término de preposición) y suelen alternar con oraciones de infinitivo y con sintagmas nominales. No supone, por tanto, un problema (re)analizarlas como sustantivas.

Sin embargo, tras explicar por qué ciertas oraciones adverbiales son en realidad sustantivas o de relativo, queda todavía un grupo importante de construcciones que no encajan en una clasificación bimembre de las subordinadas. Como veíamos antes, en la NGLE se

\footnotetext{
${ }^{13}$ En la NGLE 2009: $§ 31.13$ y 22.7 se señala la posibilidad de considerar relativos también otro tipo de unidades que no entran en la clase tradicional de las palabras relativas, como mientras o apenas. El análisis de estas palabras como conjunciones es polémico, pues desempeñan, como los relativos, una función en la oración subordinada.
} 
Tejuelo, $\mathrm{n}^{\circ} 35.2$ (2022), págs. 173-204. La subordinación en el Glosario de términos gramaticales...

habla para todas ellas de "construcciones" de valor condicional, causal, comparativo, final, etc. En el GTG no se tratan todas igual, y solo para algunas de ellas se mantiene el término de subordinada adverbial, como vamos a ver.

Ese grupo de construcciones se puede dividir a su vez en dos:

a. Construcciones que no son oracionales (es decir, no se construyen a partir de una forma verbal, aunque puedan contener una): las construcciones comparativas y consecutivas, constituyen siempre segmentos no oracionales. En un ejemplo como Esa casa es [SA más amplia que esta], el segmento entre corchetes es un sintagma adjetivo, y hay discusión entre los gramáticos sobre si el núcleo es el cuantificador más (que seleccionaría dos argumentos, llamados "diferencial" y "coda" en Brucart, 2003) y sobre si, dentro de este sintagma, hay un constituyente oracional con elipsis (más amplia que esta to es), y lo mismo se puede decir de todas las comparativas. ${ }^{14}$ Por tanto, se habla en el GTG, igual que en la NGLE, de construcción comparativa, en lugar de oración subordinada comparativa. ${ }^{15}$

Se puede decir lo mismo de las construcciones consecutivas, que aparecen asociadas a diversos tipos de sintagmas: nominales (Recibió [SN tantos premios que sus padres se emocionaron]), adjetivales (El niño estaba [sA tan sucio [que hubo que meterlo en la bañera]]), etc. De manera similar a las construcciones comparativas, las construcciones consecutivas constan de un cuantificador (tanto, tan, tal) y de una coda (un segundo argumento) que suele ser una oración subordinada encabezada

\footnotetext{
${ }^{14}$ En la gramática tradicional no parece haber instrumentos para analizar el segmento que esta, que en gramática generativa se puede considerar un sintagma conjuntivo.

${ }^{15}$ En el caso de las comparativas, también es controvertida la naturaleza coordinada o subordinada del segmento que encabeza. Como se explica en el GTG en la entrada conjunción comparativa, las conjunciones comparativas relacionan los dos términos de manera semejante a como lo hace una conjunción copulativa, y tienen algunas propiedades que las acercan a las conjunciones coordinantes, como el hecho de admitir infinitivos como término, frente a las conjunciones subordinantes: Era más prudente esperar que lanzar el ataque.
} 
por que. Para esta oración — pero no para toda la construcción consecutiva - se emplea el término subordinada adverbial consecutiva en el GTG (aunque no es evidente que no se pueda aplicar, en este caso y en el de las codas comparativas, un análisis de subordinación sustantiva).

b. Construcciones que son oracionales. Otro grupo de oraciones adverbiales de la tradición son claramente oraciones y en el GTG se tratan como oraciones subordinadas adverbiales. De ellas nos vamos a ocupar a continuación.

\section{2.- Las subordinadas adverbiales en el GTG}

El GTG comparte los presupuestos básicos de la NGLE y también deshace, en parte, el grupo de las subordinadas adverbiales. Sin embargo, por razones didácticas no se elimina completamente el grupo, es decir, se mantienen algunas oraciones subordinadas como adverbiales. El objetivo de los apartados que siguen es explicar las razones por las que se ha hecho así. Veremos que las diferencias entre las dos obras no son sustanciales, aunque el GTG retome el término de subordinación adverbial por razones didácticas que se explicarán a continuación. ¿Qué razones didácticas? Esencialmente, la no introducción de herramientas teóricas que no son necesarias en niveles preuniversitarios.

Hemos visto en el apartado anterior que hay acuerdo entre los gramáticos formales sobre la existencia de solo dos tipos de subordinación, la sustantiva y la relativa. También hemos visto cómo un grupo de las tradicionalmente llamadas subordinadas adverbiales (las propias) se puede reducir sin problema a uno de los dos grupos anteriores (las de relativo).

El GTG parte de los planteamientos de la NGLE, pero no se pierde de vista su finalidad didáctica, ya es una obra orientada a la enseñanza secundaria.

En vista de las dificultades para explicar algunas subordinadas adverbiales como sustantivas o relativas, en esta obra se opta por 
mantener la etiqueta $\mathrm{y}$, al menos parcialmente, el grupo de las subordinadas adverbiales.

En el GTG se mantiene el término de oración subordinada adverbial para aquellos segmentos que son claramente oracionales y están encabezados por una conjunción de significado causal, final, condicional, etc. (una conjunción distinta de que, que introduce sustantivas). Son oraciones que ocupan posiciones de adjunto, no de argumento, y en ellas la oración flexionada no alterna con una oración de infinitivo o con un sintagma nominal. Por ello, no se incluyen en el grupo de las subordinadas sustantivas a pesar de ir encabezadas por una conjunción.

Las oraciones subordinadas adverbiales que se reconocen en el GTG son las siguientes (indicamos con negrita las conjunciones con significado):

a. Causales: Como no venía el autobús, cogieron un taxi.

b. Modales: Hizo la tarta según explicaba la receta.

c. Temporales: Pone la tele apenas llega a casa.

d. Ilativas: Está lloviendo, así que no saldremos.

e. Condicionales: Si viene pronto, iremos al cine.

f. Concesivas: Aunque sea tarde, voy a ver la película.

g. Consecutivas: Hacía tanto frío que no fuimos al cine

Entre las construcciones de significado adverbial que son oracionales se pueden diferenciar dos grupos:

a. Oraciones que contienen la conjunción que, con diversos significados como causal (Vamos a coger el autobús, que no puedo más), final (Date la vuelta, que te vean todos), etc. Si tuviéramos un tipo de oraciones, llamadas sustantivas, que se caracterizaran por la presencia de una conjunción completiva, estas oraciones podrían entrar en ese grupo. Pero, como hemos visto, las oraciones con la conjunción completiva se caracterizan como sustantivas porque ocupan posicionales argumentales típicas de $\mathrm{SN}$, y con estos criterios funcionales, estas oraciones 
no encajarían en ese grupo. Por ello, en el GTG se mantiene para estas oraciones la etiqueta subordinada adverbial causal y final, encabezadas por una conjunción causal y final respectivamente. ${ }^{16}$

b. Oraciones encabezadas por diversas conjunciones que se caracterizan, frente a que, por tener un significado gramatical de tipo causal (Iremos a un restaurante, ya que es mi cumpleaños; Dado que es tarde, dejamos la película para mañana), condicional (Como no te calles me voy; Cuando lo dice será por algo; Iré siempre y cuando me acompañes) o concesivo (Aunque hacía calor, salieron a dar un paseo), ${ }^{17}$ etc.

Es importante señalar que estas oraciones no forman un grupo homogéneo, más allá de ocupar posiciones de adjunto. La razón por las que las tratamos aquí es que sí se caracterizan porque no se pueden reducir, de manera sencilla, a ninguno de los dos tipos de subordinadas considerados, es decir, a sustantivas o relativas. $^{18}$

Algunas de estas oraciones, para las que en el GTG se ha mantenido la etiqueta de subordinada adverbial, no son ni siquiera subordinadas, en el sentido de que no están incluidas dentro de la oración principal, sino que mantienen una relación de concatenación o

\footnotetext{
${ }^{16}$ Sin embargo, como señalábamos más atrás, no está claro que sea la conjunción la que tiene el significado causal o final. Se podría pesar que hay una sola conjunción que, la misma que aparece en las subordinadas sustantivas, y en la coda comparativa, consecutiva y superlativa. Esta conjunción podría tomar términos no oracionales, como en las comparativas, o términos oracionales.

${ }^{17}$ Creemos que la forma aunque podría incluirse en el grupo $a$ ) si se considera que, en realidad, se trata de una amalgama del adverbio escalar aun más la conjunción que (NGLE: § 47.2n-ñ, 47.12e). Desde ese punto de vista, aun seleccionaría una oración sustantiva. Es fácil comprobar que la sustantiva puede sustituirse por una construcción de gerundio, pero no de infinitivo (aunque vengas/aun viniendo/*aun venir), lo cual plantea una asimetría con respecto a lo que sucede con las estructuras causales y finales introducidas mediante por y para respectivamente.

${ }^{18}$ No es imposible defender, para algunos de estos casos (dado que, aunque, etc.), un análisis de subordinación sustantiva. Dicha posibilidad puede plantearse en el aula de Secundaria, pero el motivo por el que el GTG no explora esa opción es que requiere de una maquinaria que probablemente exceda lo que es necesario saber en niveles preuniversitarios.
} 
interordinación con la principal. Entre ellas están las oraciones condicionales y las concesivas, y algunas causales, finales o modales cuando aparecen antepuestas, o pospuestas pero separadas por una pausa de la principal.

Ya hemos visto que para mantener la intuición tradicional de que ese conjunto de elementos establece una relación de adjunción (causal, condicional, temporal, etc.) con el verbo (o con toda la oración principal), se emplea en la NGLE el término construcción, que es más neutro que oración y que permite englobar a SN, SP y SAdv de significado "adverbial" en la misma clase, sin necesidad de afirmar su naturaleza oracional. Es importante recordar que una etiqueta como construcción comparativa o construcción condicional no equivale sin más a subordinada comparativa o subordinada temporal, pues con ella se quiere señalar sobre todo una relación semántica, no un tipo de constituyente.

En resumen, la teoría gramatical actual considera que en realidad hay solo dos maneras de subordinar, y rechaza la subordinación adverbial como un tercer procedimiento de subordinación. Esa idea es robusta y puede, por tanto, llevarse a las aulas. Una parte importante de las llamadas oraciones subordinadas adverbiales no son en realidad oraciones, sino SP y SAdv adjuntos de significados "adverbiales" en el sentido de los significados que tienen tradicionalmente los complementos adjuntos. Por eso, en la NGLE se sustituye el término subordinada adverbial por construcción adverbial (condicional, concesiva, comparativa, etc.), lo que implica que tenemos una construcción — no necesariamente oracional- que tiene el tipo de significado descrito.

Otra parte son en realidad oraciones de relativo con antecedente implícito. Por último, un tercer grupo sí son oraciones. La reducción de estas últimas a los dos primeros grupos implica, entre otras cosas, una reformulación de relativas y sustantivas en otros términos y exige un conjunto de instrumentos de análisis que no suele estar disponible en las aulas no universitarias. Por todo ello, en el GTG se optó por mantener la etiqueta de subordinación adverbial para ese conjunto de oraciones. 
Sin embargo, como hemos tratado de explicar, la etiqueta esconde construcciones de muy diversa naturaleza y, por ello, quizá lo mejor, en el caso de la enseñanza no universitaria, es evitar entrar en el análisis sintáctico de estas oraciones, pues habría que hacerlo dejando de lado demasiados detalles.

\section{3.- Problemas de escritura asociados a la gramática de la subordinación}

En este apartado presentaremos algunos ejemplos de posibles ejercicios relacionados con la subordinación que tienen una aplicación directa en la escritura.

\section{EJERCICIO 1}

Observa el siguiente par mínimo y explica la diferencia de significado entre las dos oraciones (sobre esta cuestión, véanse los numerosos ejemplos recogidos en la OLE: 3.4.3.2.3):

a. Pensó en quién la había traicionado

b. Pensó en quien la había traicionado

\section{Explicación}

La reflexión que desencadena este ejercicio tiene que ver con la diferente denotación que tienen las oraciones interrogativas indirectas, por un lado, y las relativas sin antecedente expreso, por otro. Pocas veces se explica en las aulas de secundaria que la denotación de una oración es muy diferente de la de un SN. Las primeras denotan eventos, estados de cosas o situaciones, mientras que los segundos denotan entidades, entendiendo entidad sentido amplio para abarcar personas, animales, cosas, sentimientos, etc.

El interrogativo tónico de $a$ introduce una interrogativa indirecta. El significado de $a$ es 'Pensó en qué persona será la que la ha traicionado', es decir, no se sabe quién ha sido el que ha traicionado. En cambio, el hecho de tener en $b$ una relativa sin antecedente de término de la preposición hace que el significado sea muy diferente. La relativa sin antecedente se entiende que tiene el antecedente implícito o tácito, y 
es, por tanto, un sintagma nominal con una relativa ('la persona que la había traicionado'), lo que explica que su denotación sea una persona en concreto. Por ello, en $b$ se está pensando en una persona en concreto, se sabe de quién se está hablando.

Sabemos que la acentuación de interrogativos y relativos representa un problema en la escritura, sobre todo cuando se trata de interrogativas indirectas, es decir, de oraciones que no van encabezadas con el signo de interrogación. Lo que queremos señalar aquí es que reflexionar, a través de ejercicios de pares mínimos como este, sobre el diferente significado de interrogativas y relativas sin antecedente puede ayudar a mejorar este problema concreto de acentuación.

\section{EJERCICIO 2}

Observa los siguientes pares y explica las diferencias:

a. Tenía pocos años, pero demostraba mucha experiencia

b. Tenía pocos años y, sin embargo, demostraba mucha experiencia

a. *Tenía pocos años, demostraba pero mucha experiencia

b. Tenía pocos años y demostraba, sin embargo, mucha experiencia

\section{Explicación}

En los libros de texto de secundaria se mezclan a veces, de manera indiscriminada, las conjunciones y los marcadores discursivos. Sin embargo, el comportamiento sintáctico de unas y otros es muy diferente y la ortografía asociada a ellos también. Por ello, es importante que el alumno sea consciente de las diferencias sintácticas entre una conjunción que encabeza una oración y un conector discursivo, que está fuera de ella. Tanto pero como sin embargo establecen una relación de oposición entre dos elementos en el texto. Sin embargo, las conjunciones no tienen movilidad posicional, forman parte de la oración - coordinada o subordinada - que encabezan y por ello aparecen siempre en primer lugar. El conector sin embargo tiene autonomía entonativa, por lo que debe aparecer precedido y seguido de signos de puntuación en un texto escrito, y tiene mucha más movilidad. El conocimiento explícito de esta diferencia gramatical permite, por un lado, puntuar con mayor corrección y, por otro, ofrece más 
posibilidades de elección a la persona que escribe y sabe que puede situar los marcadores discursivos en distintos lugares de la oración.

\section{EJERCICIO 3}

Sustituye la secuencia subrayada por otra que contenga un sustantivo y explica qué diferencias de matiz percibes entre una y otra:

a. Salió de casa pese a que su madre se lo había prohibido terminantemente

b. Pudo llegar a tiempo gracias a que había comprado un coche el $\underline{\text { mes pasado }}$

c. La mascota murió a consecuencia de que la habían abandonado $\underline{\text { sin comida }}$

\section{Explicación}

Hemos visto que muchas expresiones de "significado adverbial", es decir, que tienen significados habitualmente asociados con los adjuntos, como la consecuencia, la causa, etc., no constituyen subordinadas adverbiales sino preposiciones o locuciones prepositivas que transmiten estos significados. Como cualquier preposición, estas expresiones aceptan términos nominales, o bien oracionales (oraciones flexionadas con que, o bien oraciones de infinitivo). Esto hace que presenten posibilidades de construcción diferentes, que se pueden asociar con diferentes fines en un escrito formal. Expresiones como pese a, gracias a o a consecuencia de se asocian más con el texto escrito que con el oral, e implican un cierto grado de formalidad con la que los estudiantes no están tan familiarizados. Las expresiones con los sustantivos correspondientes (pese a la prohibición, gracias a la compra, a consecuencia del abandono) resultan muy adecuadas, como se señala en Montolío (2014: $\S 1$ ), en los textos formales y suponen un grado importante de abstracción y generalización.

\section{EJERCICIO 4}

Observa la puntuación de las siguientes oraciones, corrígela en los casos en los que lo consideres oportuno y explica por qué lo has hecho.

a. Ella que vive cerca puede ir a buscarte

b. Azorín es un escritor el cual destaca por una prosa desnuda

c. Se subieron al coche, dentro del cual, estaban sus amigos 
d. Ayer se celebró la graduación, durante la cual, todos se emocionaron

Explicación

Este ejercicio se centra en la puntuación de las relativas especificativas y explicativas. Las oraciones de relativo, como es sabido, se dividen en dos grupos, especificativas y explicativas según el nivel estructural en el que modifican al sintagma nominal. Las especificativas modifican al sustantivo, o al sustantivo $y$ sus complementos, forman parte del sintagma nominal y por ello no se separan con coma de su antecedente. Estas relativas contribuyen a la delimitación del posible referente del sintagma nominal. Así, en una oración como Juan tiene tres hermanos que son mayores que él, sabemos que hay tres hermanos mayores que Juan, pero es posible que tenga más hermanos. Esto se deduce directamente de la estructura del sintagma nominal y del sintagma sobre el que incide el cuantificador tres: hermanos que son mayores que él. En cambio, en Juan tiene tres hermanos, que son mayores que él, la relativa explicativa es un inciso, por tanto, no interviene en la delimitación del referente del sintagma nominal. En este caso, Juan tiene únicamente tres hermanos, sobre los que se añade la precisión de que son mayores que él. Las relativas explicativas son incisos que modifican a todo el sintagma nominal en su conjunto, y no contribuyen a la especificación del referente del sintagma nominal. El hecho de una relativa especificativa contribuya a la delimitación del referente explica que la única posibilidad en $a$ sea una explicativa, pues un pronombre personal, igual que un nombre propio, señalan directamente a su referente.

El pronombre relativo complejo el cual y sus variantes de género y número es a menudo percibido como perteneciente a un registro más elevado, y por eso los alumnos a menudo lo utilizan, muchas veces de manera incorrecta, en sus textos. En el caso de $b$ los alumnos han de saber que el cual solo puede aparecer en relativas explicativas (a menos que el relativo sea término de preposición, como en Un escritor del cual he leído varios libros). En cuanto a $c$ y $d$, si los alumnos fueran conscientes de que los relativos, como cualquier elemento subordinante, están dentro de la oración y no constituyen 
incisos, no pondrían la coma tras el relativo. En todos los casos anteriores, tener conocimiento de cómo se construye el sintagma nominal y sobre cómo inciden los diferentes tipos de relativas sobre él permite puntuar las oraciones que contienen relativos con corrección.

\section{EJERCICIO 5}

Observa el siguiente par mínimo y explica la asimetría de gramaticalidad, asociada con factores ortográficos:

a. *No sé cuando vendrás

b. No sé cuándo vendrás

\section{Explicación}

La reflexión que desencadena este ejercicio tiene que ver, de nuevo, con la diferente denotación que tienen las oraciones sustantivas y relativas. El verbo saber selecciona, típicamente, una oración subordinada sustantiva en función de CD. En este caso, es una interrogativa indirecta $\mathrm{y}$, como tal, debe estar introducida por un elemento interrogativo (cuándo), no uno relativo (cuando). Una oración sustantiva funciona, tal y como hemos dicho, como un argumento (con diversas funciones sintácticas: sujeto, CD o término de preposición), pero una oración de relativo especificativa es un predicado (su función es, siempre, la de CN). Se da, por lo tanto, un problema de selección. Una manera de solucionar el problema que plantea este par mínimo es cambiar la oración de relativo por una sustantiva (cambiando cuando por cuándo).

\section{EJERCICIO 6}

Compara los pares de oraciones siguientes y explica las diferencias de significado que percibes entre ellas:

(1)a. No me dejaron entrar en el examen porque llegué cinco minutos tarde

b. Porque llegué cinco minutos tarde no me dejaron entrar en el examen (ejemplo de Galán, 1999, p. 3606)

(2)a. No podremos ir al cine, puesto que saldrás tarde del trabajo b. Puesto que saldrás tarde del trabajo, no podremos ir al cine 
(3)a. He escrito a María porque se lo había prometido

b. Escribe a María, porque se lo has prometido (ejemplo de Galán, 1999, p. 3609)

(4)a. No hemos salido porque llovía

b. No hemos salido porque lloviese

c. No porque lloviese íbamos a dejar de salir

d. No hemos salido, porque llovía

El objetivo de este ejercicio es reflexionar sobre cómo la distinta posición de los complementos afecta al significado, en concreto, nos vamos a centrar en las oraciones causales: qué diferencias de significado implica anteponer una causal, por qué a veces se separa la causal del predicado principal con una pausa o una coma (es decir, la diferencia entre causales integradas en el predicado y causales periféricas), qué diferencias hay entre los distintos nexos causales, cómo afecta la negación al significado de las causales. Este mismo ejercicio se podría plantear con otras subordinadas como las finales, consecutivas, condicionales, concesivas, etc.

En $1 a$ la oración causal introducida por porque está integrada en el sintagma verbal. Como las causales normalmente son información remática o nueva, suelen ir pospuestas al predicado principal. Cuando se anteponen, como en $1 b$, la causal se focaliza y de esta manera se enfatiza la causa (Galán, 1999, p. 3606), por eso $1 b$ se podría parafrasear como 'Solo porque llegué cinco minutos tarde no me dejaron entrar en el examen'. Si se pide a los alumnos que pronuncien las oraciones en alto, se verá que $1 b$ también se asocia con una entonación ascendente que no tiene su correspondiente de la. La anteposición de la oración, como señala Galán (1999), se convierte en un mecanismo de refuerzo de la opinión del hablante, en el sentido de que se indica que se encuentra desproporcionada la relación entre la consecuencia y la causa que lo provoca. Se ve en este ejemplo que la anteposición de la causal puede tener un valor particular que se puede emplear, por ejemplo, en un texto argumentativo. 
El contraste entre $2 a$ y $2 b$ es más sutil. La forma puesto que introduce siempre una causal periférica (en concreto, este tipo de oraciones se denominan causales explicativas), es decir, una oración no integrada en el predicado principal. Por eso, las oraciones introducidas por este nexo siempre se separan del predicado principal con una coma. La vinculación de estas causales con el predicado es menor que con las causales que están dentro del predicado principal. En estas oraciones, se enuncia la oración principal, que se considera correcta o adecuada, y luego se justifica por qué lo es (NGLE: § 46.3j). La diferente posición implica que en $2 b$ la causal introduce una información temática o conocida, mientras que $2 a$ la información es remática o nueva.

El contraste en $3 a$ y $3 b$ representa la diferencia tradicional entre causal del enunciado y causal de la enunciación (estas últimas son un tipo de causal explicativa). En el primer caso, la causal está integrada en el predicado y designa una circunstancia del predicado principal. En $3 b$, en cambio, la relación entre el predicado principal y la causal es más periférica: la causal se separa con pausa de la principal, no afecta directamente al verbo y establece una relación semántico-pragmática más amplia con el predicado principal, semejante a la que establece un adverbio oracional. Se entiende en este caso que la causal no indica la causa por la cual se ha producido lo denotado en la oración principal, sino que es una justificación de por qué se da la orden de escribir.

Por último, los contrastes de 4 tienen que ver con la interacción de la causal con la negación. En $4 a$ y $4 b$ la causal está integrada en el predicado. En $4 a$ la oración causal queda fuera del ámbito de la negación, que afecta solo al predicado principal. Se podría parafrasear como 'Es un hecho que no hemos salido y la razón es que llovía'. En $4 b$, en cambio, la negación afecta a la causal (no... porque lloviese), lo que provoca la presencia del subjuntivo. En este caso, la paráfrasis sería 'Hemos salido, pero la causa no ha sido que lloviese'. En $4 c$ la negación se antepone con la causal, de esta manera se focaliza que esta no es la causa, sino que debe de haber otra. Este puede ser, de nuevo, un mecanismo retórico que se utilice en un texto. Por último, en $4 d$ la causal es explicativa y queda fuera del ámbito de la negación, por lo 
que la presencia del subjuntivo no sería posible: *No hemos salido, porque lloviese.

\section{4.- Conclusiones}

El conocimiento explícito de la gramática puede resultar útil para tareas que vayan más allá del análisis de estructuras lingüísticas: favorecer el razonamiento abstracto, mejorar la capacidad de observación de los estudiantes, desarrollar su capacidad argumentativa y corregir problemas de escritura. Sin embargo, para ello es necesario que los profesores tengan un buen dominio de la gramática (superior al que se despliega en los libros de texto) y esto no siempre se da. Una tarea pendiente, por tanto, en la que los profesores de secundaria y los profesores de universidad podrían (y deberían) colaborar, es la creación de materiales didácticos que vinculen específicamente la gramática con la mejora de la escritura. En el caso de la creación de textos (de las dimensiones que sean), tal mejora podría basarse en las estrategias lingüísticas que permiten establecer relaciones conceptuales complejas (causa, consecuencia, condición, etc.). Ese es, precisamente, el terreno de la llamada "subordinación adverbial". Este artículo ofrece un resumen de cómo se analizan estas estructuras en la NGLE y el GTG, para a continuación plantear una serie de ejercicios que intentan hacer explícita no solo las ventajas de las propuestas de ambas obras, sino también los beneficios que pueden aplicarse en el campo de la escritura y la ortografía.

\section{Referencias bibliográficas}

Areses, I., y Pérez Jiménez, I. (en preparación). La subordinación adverbial. En Á. J. Gallego y E. Gutiérrez-Rodríguez (Coords.), La enseñanza de la gramática: teoría, metodologías y competencias. Madrid: Visor.

Bosque, I., y Gutiérrez-Rexach, J. (2009). Fundamentos de sintaxis formal. Madrid: Akal. 
Brucart, J. M. (2003). Adición, sustracción y comparación: un análisis composicional de las construcciones aditivo-sustractivas del español. En Sánchez Miret, F. (Ed.), Actas del XXIII Congreso Internacional de Lingüística y Filología Románica. Tübingen: Max Niemeyer, 11-60. http://filcat.uab.cat/clt/publicacions/reports/pdf/GGT02-4.pdf

Brucart, J. M., y Gallego, Á. J. (2016). Aspectos formales e interpretativos de la subordinación adverbial. En M. V. Pavón Lucero (Ed.), Las relaciones interoracionales en español. Berlin: De Gruyter, 161-199.

Brucart. J. M., y Gallego, Á. J. (2009). L'estudi formal de la subordinació i l'estatus de les subordinades adverbials. Llengua $i$ Literatura, 20 , 139-191. https://raco.cat/index.php/LlenguaLiteratura/article/view/304970

Camps, A., y Fontich, X. (2019). Teachers' concepts on the teaching of grammar in relation to the teaching of writing in Spain: A case study. L1 Educational Studies in Language and Literature, 19, 136. https://11.publication-archive.com/publication/1/1734

Cassany, D. (1993). La cocina de la escritura. Barcelona: Anagrama.

Escandell Vidal, M. V. (en preparación). Gramática, lengua hablada y lengua escrita. En Á. J. Gallego y E. Gutiérrez (Coords.), La enseñanza de la gramática: teoría, metodologías y competencias. Madrid: Visor.

Fontich, X., y Camps, A. (2015). Gramática y escritura en la educación secundaria: estudio de caso sobre los conceptos de los profesores. Tejuelo: Didáctica de la Lengua y la Literatura. Educación, 22,

11-27.

https://dialnet.unirioja.es/servlet/articulo?codigo=5317968

Galán, C. (1999). La subordinación causal y final. En I. Bosque y V. Demonte (dirs.), Gramática descriptiva de la lengua española, vol. 3. Madrid: Espasa, 3597-3642.

Montolío, E. (2014). Mecanismos de cohesión II: los conectores. En Manual de escritura académica y profesional, vol. II. Barcelona: Ariel.

Myhill, D., Jones, S., y Watson, A. (2013). Grammar matters: How teachers' grammatical knowledge impacts on the teaching of 
writing. Teaching and Teacher Education, 36, 77-91. (citado Myhill et al 2013).

https://www.sciencedirect.com/science/article/abs/pii/S0742051X13001 194?via\%3Dihub

Myhill, D., Jones, S., Lines, H., y Watson, A. (2012). Rethinking grammar: The impact of embedded grammar teaching on students' writing and students' metalinguistic understanding. Research Papers in Education, 27 (2), 139-166 (citado Myhill et al. 2012). https://www.tandfonline.com/doi/abs/10.1080/02671522.2011.637640

Pavón Lucero, M. V. (1999), Clases de partículas: preposición, conjunción y adverbio. En I. Bosque y V. Demonte (Dirs.), Gramática descriptiva de la lengua española. Vol. 1, Madrid: Espasa, 565-656.

Pavón Lucero, M. V. (2012). Estructuras sintácticas en la subordinación adverbial. Madrid: Arco-Libros.

RAE-ASALE (2009). Nueva gramática de la lengua española, Madrid: Espasa (obra citada como NGLE).

RAE-ASALE (2019). Glosario de términos gramaticales. Salamanca: Universidad de Salamanca (obra citada como GTG).

Rojo, G. (1978). Cláusulas y oraciones. Verba, Anejo 14. Santiago de Compostela: Universidad de Santiago de Compostela.

Rojo, G. (1983). Aspectos básicos de sintaxis funcional. Málaga: Librería Ágora. 